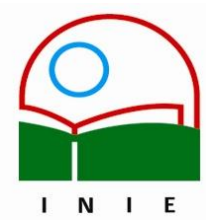

Actualidades Investigativas en Educación

Revista Electrónica publicada por el

Instituto de Investigación en Educación

Universidad de Costa Rica

ISSN 1409-4703

http://revista.inie.ucr.ac.cr

COSTA RICA

\title{
LA ENSEÑANZA DE LA LENGUA ESPAÑOLA EN I Y II CICLOS DE LA EDUCACIÓN GENERAL BÁSICA. APORTACIONES DE LA UNIVERSIDAD DE COSTA RICA
}

THE EDUCATION OF THE SPANISH LANGUAGE IN I AND II CYCLES OF BASIC THE GENERAL EDUCATION. CONTRIBUTIONS OF THE UNIVERSITY OF COSTA RICA

Volumen 7, Número 3

pp. 1-35

Este número se publicó el 15 de diciembre 2007

Marielos Murillo Rojas

Mauren Morales Rivera

La revista está indexada en los directorios:

LATINDEX, REDALYC, IRESIE, CLASE, DIALNET, DOAJ, E-REVIST@S,

La revista está incluida en los sitios:

REDIE, RINACE, OEI, MAESTROTECA, HUASCARAN 


\title{
LA ENSEÑANZA DE LA LENGUA ESPAÑOLA EN I Y II CICLOS DE LA EDUCACIÓN GENERAL BÁSICA. APORTACIONES DE LA UNIVERSIDAD DE COSTA RICA
}

THE EDUCATION OF THE SPANISH LANGUAGE IN I AND II CYCLES OF BASIC THE GENERAL EDUCATION. CONTRIBUTIONS OF THE UNIVERSITY OF COSTA RICA

\author{
Marielos Murillo Rojas ${ }^{1}$ \\ Mauren Morales Rivera ${ }^{2}$
}

\begin{abstract}
Resumen: Este artículo expone los principales hallazgos de las investigaciones que, de 1990 al año 2007, se han desarrollado en la Universidad de Costa Rica, particularmente en la Facultad de Educación, sin obviar el aporte de otras instancias de la universidad, en relación con la enseñanza y el aprendizaje de la lengua española en la educación primaria costarricense.
\end{abstract}

Palabras claves: ENSEÑANZA/ APRENDIZAJE/ LENGUA ESPAÑOLA/ EDUCACIÓN PRIMARIA/

\begin{abstract}
This article exposes the main findings of the investigations that, of 1990 to year 2007, have been developed in the University of Costa Rica, particularly in the Faculty of Education, without avoiding the contribution of other instances of the university, in relation to the education and the learning of the Spanish language in the Costa Rican primary education.
\end{abstract}

Keywords: EDUCATION/ LEARNING/ SPANISH LANGUAGE/ PRIMARY EDUCATION/

\section{Introducción}

"Lo que fue funcional a un sector social, y a los objetivos que en un momento histórico se formularon, no lo es hoy para todos los niños y para los objetivos que actualmente nos planteamos" (Costedo, 1996).

Actualmente vivimos en un mundo caracterizado por el movimiento y las transformaciones, un mundo que impone nuevos retos al sistema escolar global, principalmente en cuanto a su misión alfabetizadora, esa misión que está en las raíces de su función social. Hoy, más que nunca, es necesario realizar investigaciones en cuanto a la enseñanza y el aprendizaje de la lengua española, investigaciones que sirvan como fundamentos para adecuar o reestructurar las actuales prácticas educativas.

\footnotetext{
${ }^{1}$ Doctora en Formación de Profesorado área Didáctica de la Lengua Española, de la Universidad de Extremadura, España. Máster en Lingüística; Licenciada en Educación Preescolar, ambos títulos de la Universidad de Costa Rica. Profesora de la Escuela de Formación Docente, Universidad de Costa Rica. Correo electrónico: marielosmuro@yahoo.es

${ }^{2}$ Licenciada en Educación Primaria de la Universidad de Costa Rica. Actualmente es maestra en la Escuela Omar Dengo. Correo electrónico: maurenmr@yahoo.com
}

Artículo recibido: 20 de agosto, 2007

Aprobado: 12 de diciembre, 2007 
En vista de lo señalado, este artículo tiene como objetivo fundamental revisar los principales hallazgos de las investigaciones que, desde 1990 al año 2007, se han realizado en la Universidad de Costa Rica, particularmente en la Facultad de Educación, sin obviar el aporte de los Institutos de Investigación (INIE e INIL) y de las revistas especializadas.

Por tanto, se parte de la revisión y lectura cuidadosa de las siguientes fuentes:

- Los listados de los trabajos finales de graduación publicados en la Facultad de Educación de la Universidad de Costa Rica, a partir de la década del noventa, con el fin de seleccionar aquellas relacionadas con el área de español en educación primaria -competencias lingüísticas de los escolares, diagnósticos y propuestas para la enseñanza de los diferentes componentes de la lengua-.

- Las investigaciones producidas en los Institutos de Investigación de la Universidad de Costa Rica: Instituto de Investigaciones en Educación (INIE) e Instituto de Investigaciones Lingüísticas (INIL), a partir de 1990 y que conciernen al área de español en educación primaria.

- Todos los artículos publicados en relación con la enseñanza y el aprendizaje de la lengua española en educación primaria en las revistas de la Universidad de Costa Rica: Revista Káñina: Revista de Artes y Letras, Revista Educación, Revista de Filología y Lingüística y Revista Actualidades en Educación.

Este ensayo se organiza en tres partes: aportes de los trabajos finales de graduación, aportes de los institutos de investigación y publicaciones en revistas especializadas.

\section{A. Aportes de los trabajos finales de graduación}

De todos los trabajos finales de graduación (para optar por el grado de licenciatura o máster y en un caso, por el doctorado) que se han publicado en la Universidad de Costa Rica, desde 1990 hasta la fecha, solamente veinte de estos se relacionan con la enseñanza y el aprendizaje de la lengua española en I y II ciclos de la Educación General Básica.

Para empezar, existen tres tesis sobre la formación de docentes en educación primaria, tema que resulta relevante para este artículo, pues la práctica educativa en las escuelas depende en gran medida de la formación inicial de los educadores. 
En 1990, Olimpia López presentó un "Análisis contextual de los planes de estudio para la formación de docentes de enseñanza primaria en la Facultad de Educación de la Universidad de Costa Rica", que determina las variaciones de los planes para la formación de docentes de primaria desarrollados por la Universidad de Costa Rica entre 1960 y 1987; y establece cómo perciben los profesores la calidad de su formación en relación con su práctica como docentes, de acuerdo con los diferentes planes de estudio. De esta investigación se concluyó que existe poca vinculación entre las políticas y concepciones del Ministerio de Educación Pública ${ }^{2}$ y de la Facultad de Educación y que no hay claridad curricular en cuanto el perfil de maestro que se desea formar.

También en 1990, la investigación de Alicia Sequeira, titulada "La formación de educadores en educación primaria ante la demanda del MEP”, clarifica los criterios orientadores de la demanda de educadores del MEP, hecha entre 1986 y 1987, a las universidades estatales del país y presenta los principios curriculares de la respuesta de las instituciones formadoras de educadores de educación primaria. En esta investigación se constató que las tres universidades estatales improvisaron los planes de formación docente con enfoques curriculares poco claros y perfiles de salida descontextualizados.

Y siete años después, Melida Carballo y Nuria Mairena (1997) realizaron una "Evaluación curricular del plan de bachillerato en educación primaria de la Sede de Guanacaste", con el fin de evaluar la congruencia que existe entre la oferta educativa del Bachillerato en Educación Primaria y las demandas del medio sociocultural de la región. De esta investigación, se concluyó que el plan de bachillerato es débil en valores, que tiene $80 \%$ de viabilidad en sus objetivos, que sus contenidos son repetitivos y conductuales, que no incorpora las necesidades y los problemas del contexto regional y que no prepara para el desempeño en zonas rurales y en escuelas unidocentes. Por lo tanto, existe desfase entre la política educativa y el programa de carrera.

Así, a partir de dichos estudios, se concluye que hay deficiencias en los programas de formación de los docentes de primaria, ya que los planes de estudio de la Facultad de Educación están desvinculados de las concepciones del MEP y carecen de contextualización regional y viabilidad. Por consiguiente, podría inferirse que tampoco hay claridad en el

${ }^{2}$ En adelante MEP 
enfoque de la enseñanza y del aprendizaje de la lengua española en la educación primaria costarricense, pues ni siquiera parece estar claro el perfil de maestro que se desea formar.

Por otra parte, se han realizado dos estudios en relación con el rendimiento académico general de los estudiantes -incluye la asignatura de español-.

El primero se titula "Estudio del rendimiento académico de los alumnos de sexto año de Educación General Básica de la dirección regional de San José”, de Ana Cristina Meza (1994), el cual analiza el impacto que algunos factores extrínsecos e intrínsecos relacionados con el proceso educativo tienen en el rendimiento académico de los estudiantes de sexto año. A partir de los resultados obtenidos se concluye que el rendimiento académico de los estudiantes está condicionado por la formación profesional y el ingreso económico de los docentes, el nivel educativo y socioeconómico de los padres y el tipo de escuela. Es importante resaltar que se habla de rendimiento académico en general y, en el caso del español, no se especifica cuál o cuáles serían las áreas deficitarias, información inestimable para realizar algún tipo de propuesta que revierta la situación existente.

La segunda investigación, "Análisis de algunos factores que incidieron en el fracaso escolar de los alumnos reprobados en cuarto grado de la Dirección Regional de Educación de San Ramón en 1996", desarrollada por Campos, Ledesma, Rivera y Villalobos (1998) concluyó que la sobreedad cronológica y el desinterés de los estudiantes, el bajo nivel educativo de los padres, la desintegración familiar y la amenaza de otros problemas sociales, la cantidad de contenidos curriculares y la enseñanza tradicional de los docentes son los factores que inciden negativamente en el rendimiento escolar de los niños y niñas.

De ambas investigaciones concluye que el desempeño escolar está condicionado por las características de cinco componentes educativos: docentes (práctica educativa), estudiantes (grado de motivación), padres y encargados (nivel educativo y socioeconómico), comunidad (problemas sociales, demandas, etc.) y programas (sobrecarga de los contenidos curriculares).

En 1998, Fuentes, De la O, Ortega y Zamora, publicaron un trabajo final de graduación sobre "Ventajas y desventajas del trabajo por áreas o materias de estudio, según opiniones de niños, docentes, padres de familia y autoridades, en las escuelas privadas e instituciones 
técnicas públicas del circuito 02, Liberia". Entre los principales resultados de esta investigación descriptiva están la falta de capacitación y coordinación entre los docentes, pero como contraparte se determinó que estos trabajan con más gusto debido a la mayor disposición de tiempo para planear y elaborar materiales, lo cual beneficia de uno u otro modo a los estudiantes y sus padres. Por lo tanto, este diagnóstico evidencia que a los docentes de II ciclo de EGB les gusta planear dos asignaturas -en vez de cuatro como en primer ciclo-, pero lamentablemente el trabajo por áreas conduce hacia una labor más individualista, en la que se desatiende el desarrollo integral de la gestión curricular.

En el año 2005 se presentó una investigación cualitativa sobre "Lineamientos metodológicos para la integración del tema transversal -vivencia de los derechos humanos- para la democracia y la paz y la enseñanza del español en quinto año de la EGB”, de Melba Fallas, en la cual se analizó la forma en que dicho eje transversal se incorpora en las lecciones de español de quinto grado. Tal estudio evidenció que los docentes no comprenden los temas transversales y, por lo tanto, no los integran en el proceso de enseñanza-aprendizaje de la lengua sino que los estudian como contenidos aislados mediante técnicas tradicionalistas. A partir de dichos resultados se diseñó una propuesta metodológica para integrar el tema transversal "vivencia de los derechos humanos- para la democracia y la paz" en la enseñanza y el aprendizaje de la lengua española. No obstante, la integración de dicho eje transversal pudo haber sido en cualquier asignatura, por lo que el aporte de este estudio no corresponde precisamente al área de español.

Así, los dos últimos trabajos finales de graduación expuestos se refieren a temas (trabajo por áreas e integración de un tema transversal) que no atañen específicamente a la enseñanza y el aprendizaje de la lengua materna.

Además, se han escrito dos estudios relacionados con la comprensión y la promoción de la lectura, respectivamente.

En 1996, Benavides, Brenes, Coto, Espinoza, Ibarra y Rojas, presentaron la memoria del seminario de graduación "Competencia en la comprensión de lectura de escolares que finalizan la Educación General Básica”, en la cual se determinó el nivel de comprensión de lectura de todos los estudiantes de sexto grado pertenecientes a treinta escuelas públicas de la Región Educativa de San José. Como principales conclusiones se encontró que el sexo y 
el tipo de escuela son factores determinantes en la comprensión de lectura; los docentes no ven la lectura como proceso sino como producto y usan métodos conductistas para "enseñarla"; los estudiantes rechazan las actividades propuestas por los docentes para la comprensión de lectura, visitan poco las bibliotecas y prefieren ver televisión antes que leer. Por lo tanto, la mayoría de estudiantes no alcanzan los niveles de comprensión de lectura esperados.

En el 2004, la investigación "La animación y promoción de la lectura por medio de la caja viajera como un recurso de comunicación entre la escuela y el hogar", de Barquero, Calderón, Díaz, Porras y Vega; fijó como objetivo principal promover hábitos de lectura por medio de la caja viajera -maletín con libros de literatura- y sesiones de talleres, como recursos de interacción entre el centro educativo y el hogar. Como logros de esta investigación-acción, la docente cambió su percepción con respecto a la lectura, los padres mejoraron sus hábitos lectores y los estudiantes fortalecieron su comprensión de lectura.

En suma, el primer estudio evidencia problemas asociados con la comprensión de lectura y el segundo presenta alternativas para promover la presencia de lectores competentes en las aulas.

Los siguientes tres trabajos finales de graduación también se abarcan el área de lectura, pero integran la expresión escrita.

En 1994, el trabajo final de graduación "Lectura creadora: una propuesta para incrementar las habilidades de comprensión lectora y expresión escrita”, de Bonilla, Prendas, Monestel y Porras, tuvo como principal propósito determinar si la aplicación del planteamiento didáctico denominado "Propuesta de lectura creadora" mejora las habilidades de comprensión lectora y de expresión escrita. En este se concluyó que la lectura creadora promovió las habilidades de comprensión de lectura (vocabulario, estructura de la oración, ortografía, recursos literarios, riqueza de ideas) y las habilidades de expresión escrita. Además, se comprobó que la integración de las áreas de lectura y escritura produce mejores resultados que cuando se tratan por separado.

En el 2006, el trabajo final de graduación titulado "La lectura interactiva como medio para incrementar habilidades de comprensión de lectura y expresión escrita”, de Fajardo, López, 
Montoya, Romero, Salas y Zumbado; analizó las habilidades de comprensión de lectura y expresión escrita en estudiantes de tercer año de Educación General Básica. Como conclusiones se señala que los estudiantes descodifican con fluidez, pero no releen, no infieren y no identifican ideas en el texto. Además, escriben textos con poca claridad y sin ortografía. Con respecto a las estrategias didácticas empleadas por los docentes, no estimulan el trabajo en grupo, suelen basarse en textos narrativos y usan con mucha frecuencia la pizarra, los libros y los cuadernos. A partir de dichos resultados se hizo una propuesta para favorecer la comprensión de lectura y escritura mediante la lectura interactiva.

Y en el 2000, se presentó el trabajo final "La aventura de leer y escribir", de Giselle Quirós, que identificó las prácticas pedagógicas de los docentes de primer ciclo de EGB con respecto a la promoción de la lectura y la expresión escrita. En este, se concluyó que la práctica docente es tradicional (conductista), ya que los docentes no emplean estrategias de promoción de lectura.

De estas investigaciones se infiere que en Costa Rica, la lectura se enseña y aprende tradicionalmente, como producto y no como proceso, por lo que la mayoría de escolares descodifican con fluidez, pero sin alcanzar los niveles de comprensión de lectura esperados. Por eso, se recomienda incentivar la lectura creativa y la lectura interactiva en el aula para promover la lectura y mejorar su comprensión.

En cuanto a la enseñanza y el aprendizaje de la expresión escrita se han escrito tres trabajos.

Primero, en el 2004, Susana Lukas y Xinia Granados presentaron una investigación sobre la "Aplicación de técnicas didácticas y su incidencia en el aprendizaje de la redacción de los niños y niñas de tercer grado de las escuelas Atilia Mata Freses y Santa Eduviges del cantón Central de Limón, 2004", en la que se investigó la incidencia que tienen las técnicas utilizadas por los docentes en el aprendizaje de la redacción de los estudiantes de tercer grado de dichas escuelas. En esta se constató que los docentes emplean técnicas tradicionales en la enseñanza de la producción escrita y acostumbran revisar únicamente la ortografía y la caligrafía de las redacciones sin utilizar ningún tipo de escala para hacerlo. 
Después, en el 2005, en la investigación "La expresión escrita: un medio para explorar la vida cotidiana de los niños y las niñas de tercer grado", de Jenny Chacón, se exploró la vida cotidiana de los niños de tercer grado de una escuela pública del país, mediante el análisis semántico de la expresión escrita. A partir de esta se constató que tanto en los temas asignados como en los temas libres de redacción, los niños escriben sobre acontecimientos importantes en sus vidas cotidianas; razón por la que la expresión escrita debería aprovecharse para explorar los problemas que afectan a los estudiantes. Por lo tanto, no se estudia la competencia de los escolares en la expresión escrita, sino esta como un medio para obtener información de otro tipo (socioemocional).

Y en el 2007, Castrillo, Morales, Ocampo, Pacheco y Wong; presentaron su investigación sobre "El empleo de la puntuación en la educación primaria costarricense". De esta se concluyó que: la enseñanza de la lengua materna se desarrolla de manera fragmentada, pues la escuela no promueve los espacios de aprendizaje oportunos para el estudio integral de las cuatro áreas de la lengua; hay incoherencia conceptual y metodológica en el programa de estudios de español en cuanto el abordaje de los contenidos de puntuación, por lo que no existe una metodología explícita sobre la enseñanza de los signos de puntuación en educación primaria; y la puntuación de los textos narrativos tiende a incrementarse en la medida que aumenta el nivel escolar y el nivel de completud de las historias. No obstante, a diferencia de otros trabajos finales, además de señalar la realidad educativa ofrece lineamientos metodológicos para la enseñanza de la puntuación en cada nivel de educación primaria, iniciando en primer grado con los signos de puntuación externa para concluir en sexto grado con todos los signos de puntuación interna, trabajados de manera escalonada durante toda la escuela primaria.

Por consiguiente, dichos trabajos finales de graduación diagnostican, respectivamente, las técnicas didácticas que se emplean para enseñar redacción en la escuela costarricense, la producción de redacciones como medio para conocer a los estudiantes y el uso de la puntuación; mediante pruebas de ejecución correspondientes a producciones textuales. Sin embargo, solamente la investigación sobre puntuación propone lineamientos para mejorar los puntos débiles en expresión escrita, las otras dos se limitan a la exposición de realidades.

En la Universidad de Costa Rica también se han publicado dos trabajos finales de graduación sobre el vocabulario que dominan los niños y las niñas en educación primaria. 
Ambos trabajos se basan en producciones orales y escritas. El primero solo diagnostica la disponibilidad léxica, el segundo ofrece un léxico meta para cada grado escolar.

En 1997 Cortés, González, Monge, Quesada y Washington,; publicaron su investigación "Crecimiento de la disponibilidad léxica en estudiantes de la zona de Turrialba de nivel preescolar y primer ciclo de la Educación General Básica”. En este estudio se concluye que los varones aventajan a las mujeres en cuanto al número de palabras agregadas, la zona rural supera a la urbana en cuanto al crecimiento léxico y que el patrón de crecimiento del vocabulario tiende a subir de preescolar a primer grado y de segundo a tercer grado, mientras que avanza muy lentamente de primero a segundo grados. En vista de estos resultados las investigadoras recomiendan que deben revisarse las estrategias metodológicas para la enseñanza del vocabulario, básicamente en primer grado, dados los resultados obtenidos en este estudio.

Y en el 2006, se publicó la investigación "Léxico meta para la escuela costarricense: esbozo de una propuesta metodológica para el desarrollo de la madurez léxica”, de Marta Rojas, la cual determinó un léxico para enseñar en cada año de la educación primaria. Así, se ofreció al país un léxico meta producto de la investigación, que se sustenta en la noción de lengua como variedad y en criterios de competencia educativa y de aprendizaje significativo que incorpora el juego, los valores y la creatividad para favorecer la comprensión y la producción de diferentes tipos de textos.

Por otra parte, se han presentado dos estudios sobre la filosofía del lenguaje integral. Ambos diagnostican la realidad educativa de aula y luego aplican planes de lenguaje integral para descubrir, en la evaluación final, que este nuevo enfoque produce mejores resultados en la adquisición de la lengua materna respecto de la enseñanza tradicional.

En el 2000, Marcela Aguilar culminó la práctica dirigida "El lenguaje integral: Una experiencia con niños en riesgo social", en la cual se aplicó la filosofía del lenguaje integral en la enseñanza de la lectoescritura a un grupo de seis niños en riesgo social, acogidos por el programa de formación escolar básico del centro de integración diurna del Patronato Nacional de la Infancia. Tal investigación demostró avances de los alumnos en cuanto a sus habilidades lectoras, y evidenció los beneficios del uso de la computadora para mejorar la expresión escrita de los estudiantes, así como la expresión y la comprensión orales. 
Y en el 2007, la investigación titulada "La implementación de la filosofía del lenguaje integral para el desarrollo de la lectoescritura en un grupo de la Educación General Básica”, de Aguilar, Barboza, Barth, Montero, Peña y Zúñiga; se propuso aplicar el uso de estrategias de enseñanza-aprendizaje fundamentadas en la filosofía del lenguaje integral, que favorecieran el desarrollo de la lectoescritura. Entre los principales hallazgos se encontró que la integración de contenidos favorece el alcance de objetivos didácticos planificados, que el desempeño en expresión y comprensión orales fue efectivo, que los estudiantes escriben mejor cuando tienen deseos de compartir sus escritos y que los estudiantes interactúan más cuando eligen sus lecturas.

Por último, Marielos Murillo (2004.) en la tesis doctoral "Estudio sobre el lenguaje de los escolares costarricenses: El léxico básico. La ortografía y sus características" determina el vocabulario que corresponde al léxico básico escolar -códigos oral y escrito- e identifica el vocabulario cacográfico y los patrones ortográficos seguidos por los escolares costarricenses. Por tanto, se identifican los errores más frecuentes y sus principales características; además, se propone una lista reducida de reglas que resolverían los errores ortográficos en que incurrieron repetidamente los niños costarricenses. Este estudio se basó en pruebas de ejecución y brinda un aporte significativo a la enseñanza de la lengua española en la escuela costarricense, específicamente en relación con la ortografía del léxico básico de los estudiantes.

A manera de síntesis, se puede decir que de los veinte trabajos finales de graduación que se han desarrollado en la Universidad de Costa Rica en relación con la enseñanza y el aprendizaje de la lengua española en educación primaria, el 65\% (trece) trata sobre las áreas de lectura y escritura; el 15\% (tres) tratan sobre la formación inicial de los docentes; el $10 \%$ (dos) expone el rendimiento académico de los estudiantes de primaria y otro 10\% (dos) presenta la forma en que se trabaja la asignatura de español. Por lo tanto, la expresión oral y la escucha no han sido objeto de estudio todavía en esta línea de trabajos.

También, resulta importante señalar que el 70\% (catorce) de los trabajos finales de graduación comentados se basan en pruebas de ejecución realizadas a los sujetos de investigación, pero el resto (seis) se fundamentan únicamente en las opiniones de docentes, estudiantes y otros informantes, obtenidas mediante cuestionarios y entrevistas. 
Además, el mismo porcentaje de estudios son diagnósticos, por lo que se limitan únicamente a señalar las fallas en los procesos educativos y solo seis, o sea, el $30 \%$ expone lineamientos para mejorar los puntos débiles y reforzar los puntos fuertes señalados en esos diagnósticos.

Así, de los resultados de los trabajos finales de graduación expuestos hasta el momento se puede inferir que:

- Los educadores carecen de formación básica para afrontar con éxito los retos que demanda la educación primaria, pues los programas de formación docente de las universidades estatales no tienen claridad del perfil de maestro que requiere la sociedad costarricense.

- El rendimiento académico de los escolares es deficiente en la asignatura de español en general, debido a las prácticas educativas tradicionales que no estimulan el desarrollo de competencias comunicativas.

- La mayoría de estudiantes no tienen hábitos de lectura ni alcanzan los niveles de comprensión esperados en esta área lingüística, pues en la escuela primaria se reproduce una concepción simplista de la lectura (vista como producto y no como proceso) y no se implementan actividades que la promuevan.

- La mayoría de escolares costarricenses demuestran deficiencias en la producción de textos escritos debido al empleo de estrategias metodológicas tradicionales en la enseñanza formal de la lengua española.

- Las escasas e incipientes experiencias educativas que se realizan en el país con base en la filosofía del lenguaje integral -propia del enfoque comunicativo de la lengua-, favorecen el desarrollo de las cuatro macrohabilidades de esta área lingüística lectura, escritura, habla y escucha- y sus respectivas microhabilidades.

- Finalmente, no se han realizado estudios específicos sobre las competencias orales comprensión y expresión- en los escolares; hecho que, en alguna medida, refleja la poca integración en los procesos específicos objeto de estudio en el área de lengua en la educación primaria.

Finalmente, cabe mencionar que las siguientes investigaciones se encuentran en desarrollo:

- "La lectura interactiva como medio para incrementar habilidades de comprensión de lectura y expresión escrita en II ciclo", cuyo propósito es analizar las habilidades de 
comprensión de lectura y expresión escrita en estudiantes de II ciclo de Educación General Básica.

- "La expresión escrita en la educación primaria costarricense", la cual se plantea como objetivo fundamental conocer las competencias comunicativas de los estudiantes en el área de escritura para proponer lineamientos metodológicos que mejoren su enseñanza-aprendizaje en la escuela.

\section{B. Aportes de los Institutos de Investigación: INIE e INIL}

En la Universidad de Costa Rica existen dos institutos de investigación que han desarrollado importantes proyectos sobre la enseñanza del español en I y II ciclos de la Educación General Básica: el Instituto de Investigaciones Educativas ${ }^{3}$ (INIE) y el Instituto de investigaciones Lingüísticas ${ }^{4}$ (INIL).

En el INIE, Juan Manuel Esquivel ha publicado cuatro diagnósticos sobre el rendimiento académico en general de los estudiantes de educación primaria.

En 1990, publicó el proyecto "Medición de la calidad de la enseñanza”, en el que se realizó una revisión bibliográfica sobre los fundamentos teóricos requeridos para confeccionar pruebas que midieran los conocimientos básicos en las distintas asignaturas de la educación costarricense. Los principales hallazgos en cuanto a la asignatura de español permiten afirmar un bajo rendimiento académico, tanto en lo formativo como en el dominio de objetivos, el cual varió de un año a otro y de acuerdo con la zona (rural o urbana) y la modalidad de las instituciones.

En 1994 presentó el informe final de otra investigación titulada "Medición de la calidad de la educación". En general, el estudio arrojó un bajo rendimiento en español aunque los puntajes de esta materia son ligeramente más altos que en matemáticas. También evidenció que la escolaridad de las madres, la existencia de un proyecto educativo en la institución y la buena formación del docente favorecen el rendimiento académico de los alumnos.

\footnotetext{
${ }^{3}$ En adelante INIE
}

${ }^{4}$ En adelante INIL 
Además, este mismo autor publicó en 1995 un "Informe ejecutivo de la administración de las pruebas de diagnóstico de español y matemáticas abril-mayo, 1995”. De manera general, se concluyó que los resultados de las pruebas de español fueron deficientes. No obstante, evidencian una mejoría tanto en el rendimiento general como en el dominio de conocimientos específicos en comparación con los resultados de pruebas anteriores.

Y en 1996, Juan Manuel Esquivel presentó un nuevo informe titulado "Informe político del desarrollo, validación y aplicación de las pruebas de diagnóstico", en el cual los resultados generales muestran un nivel de razonamiento y conocimiento muy bajo en todos los niveles. Los objetivos dominados pertenecen a la categoría de comprensión, es decir, se quedan en un nivel mínimo de conocimiento, básicamente en un nivel operatorio. Se continúa la tendencia de a mayor escolaridad menos rendimiento, las instituciones privadas superan a las públicas, así como las de la Región Central a las otras regiones del país, las escuelas grandes a las pequeñas, los colegios académicos diurnos a los nocturnos y técnicos, y la zona urbana a la rural. En español, específicamente, se hizo patente un bajo rendimiento.

Estos cuatro diagnósticos evidencian el problema de bajo rendimiento académico de los estudiantes de primaria en el área de español, pero no especifican cuáles son las macrohabilidades lingüísticas -lectura, escritura, expresión oral y escucha- en que los escolares muestran menor competencia; en consecuencia, es difícil tomar estos datos como insumo para reformular la programación lingüística escolar en áreas específicas.

Por otra parte, en 1990, Aurora Bogantes presentó un diagnóstico basado en pruebas de ejecución de los informantes denominado "Dominio de contenidos programáticos mínimos de matemáticas, ciencias, español, estudios sociales y actitud hacia la docencia de los aspirantes que solicitan ingresar a los planes de formación de los docentes, mediante el convenio Ministerio de Educación Pública-Universidades: Nacional, Estatal a Distancia, de Costa Rica (1989)", el cual determinó en qué medida los aspirantes a los planes de formación de las universidades estatales dominan el perfil cognitivo de II ciclo en las asignaturas básicas. Específicamente, en español se concluyó que la ortografía y la caligrafía son las áreas más críticas y por el contrario, gramática y la comunicación son las áreas de mayor dominio para los docentes en formación. 
Además, Marta Rojas y Víctor Sánchez han presentado investigaciones sobre el léxico de escolares, las cuales se basan en pruebas de ejecución de los niños y las niñas de educación primaria y generan propuestas a partir de los resultados obtenidos.

La primera investigación, publicada en el año 2003 y titulada "Desarrollo de la madurez léxica: propuesta metodológica" ofrece estrategias metodológicas y materiales didácticos adecuados para la adquisición de la madurez léxica en estudiantes de sexto año de la escuela costarricense. Además, como resultado adicional se ofrece un vocabulario de frecuencias y un listado del léxico por enseñar (léxico meta), según su orden alfabético y algunas muestras del tipo de material didáctico que se espera desarrollar en un futuro proyecto.

La segunda investigación, publicada en el 2005, "Léxico básico escolar costarricense: estudio de frecuencias"; determina el léxico básico escolar costarricense, seleccionando las palabras según frecuencia de uso en muestras escritas. El mayor beneficio que se obtuvo fue la obtención del vocabulario como instrumento de trabajo y no como fin en sí mismo. Los parámetros se presentaron en forma general, según niveles (cuarto y sexto), zona (rural y urbana) y sexo; no quieren alarmar, sino sentar las bases referenciales para abordar el problema de la pobreza léxica, dada la relación íntima que este componente tiene con el desarrollo conceptual del escolar.

Por otra parte, Marta Rojas (2007) presenta el proyecto en ejecución "Propuesta metodológica para el desarrollo de la madurez léxica: la elaboración de material didáctico para la incorporación del léxico meta", cofinanciado por la Universidad de Costa Rica y la Fundación CRUSA, cuyo propósito es ofrecer material didáctico específico para enriquecer el vocabulario y, en consecuencia, desarrollar la madurez léxica de los niños de primero a sexto grados de la educación primaria costarricense.

En síntesis, el $62.5 \%$ (cinco) de estas investigaciones realizadas en el INIE son diagnósticos y en su mayoría no generan propuestas para mejorar la enseñanza y el aprendizaje del español en educación primaria. Además, consideran esta asignatura de manera muy general, por lo que no detallan las áreas o macrohabilidades -lectura, escritura, escucha y expresión oral- que contemplan ni las competencias lingüísticas que deben reforzarse en los estudiantes. 
Por otra parte, en el INIL se encuentran tres investigaciones (una concluida y dos en desarrollo) en relación con la enseñanza de la lengua materna en la educación primaria.

En el 2006, Víctor Manuel Sánchez y Marielos Murillo presentaron el informe final de investigación, titulado "Análisis sintáctico de textos orales y escritos de niños preescolares y escolares costarricenses. Fundamento para la programación del aprendizaje lingüístico escolar", en el cual se estableció el grado de madurez sintáctica y se identificó la tipología de las estructuras sintácticas empleadas por los sujetos investigados mediante el análisis de textos orales y escritos, con el fin de orientar la enseñanza de la lengua como objeto de conocimiento e instrumento de comunicación, específicamente en el comportamiento sintáctico. Así, se concluyó que una propuesta en la enseñanza de la lengua materna en la educación primaria debe definir los objetivos que guían el proceso de enseñanza-aprendizaje y debe detallar las habilidades que ha de desarrollar el alumno en cada área. Por lo tanto, la enseñanza de morfosintaxis debe abordarse a partir del uso, de la comprensión y la producción de textos orales y escritos.

El programa de investigación "Estudios de Lexicografía -ELEXHICÓS-", bajo la dirección del Dr. Sánchez Corrales, catedrático de la Universidad de Costa Rica, está elaborando un diccionario escolar, dirigido a los niños de educación primaria costarricense. Esta obra forma parte de una serie que comprende los tres niveles de la educación costarricense: preescolar, primaria y secundaria. Por otra parte, es justo acotar que en el seno de este programa se desarrollan actividades académicas orientadas hacia la formación en la enseñanza de los diferentes componentes de la lengua; con ese objetivo se han organizado conferencias, coloquios y cursillos dirigidos a maestros y profesores de español. Igualmente se mantiene un servicio abierto de consultas sobre el español de Costa Rica y la lexicografía didáctica.

Además, cabe mencionar que el INIL tiene en desarrollo el proyecto "Del léxico fundamental de los niños preescolares y escolares costarricenses al léxico meta y su enseñanza", de Marielos Murillo, investigadora principal y Víctor Manuel Sánchez, investigador asociado. Esta investigación pretende identificar los léxicos fundamentales de los niños preescolares y de los escolares costarricenses, con el fin de determinar el léxico meta y su enseñanza. Una vez inventariados los léxicos meta de cada nivel educativo se ofrecerá una propuesta graduada para la enseñanza del léxico de cada uno de los niveles analizados. 
Por consiguiente, los tres proyectos de investigación del INIL, relacionados con la enseñanza de la lengua en educación primaria, centran su interés en el léxico de los estudiantes. Además, cabe señalar que se basan en pruebas de ejecución (textos orales y escritos) y hacen propuestas específicas para mejorar el desarrollo de competencias lingüísticas en los escolares.

Asimismo, resulta valioso señalar que la Universidad de Costa Rica tiene un proyecto de acción social titulado "El rincón de cuentos, un lugar para libros, sueños y esperanzas”, cuyo proponente y coordinador es Carlos Rubio Torres (2007). Dicho proyecto tiene el objetivo general de contribuir al desarrollo integral de la niñez, por medio del Rincón de Cuentos, en un marco multicultural, despertando en ella los hábitos de la lectura, facilitándole el acceso a libros de literatura infantil de alta calidad que estimulen y desarrollen sus capacidades de imaginar, crear y soñar.

En resumen, los principales hallazgos de los proyectos de investigación realizados en los Institutos de Investigación INIE e INIL, en el área de enseñanza del español en la educación primaria son:

- Los estudiantes de educación primaria costarricense demuestran un bajo rendimiento académico en el área de lenguaje, pues no dominan los objetivos y contenidos del programa de estudios de español.

- La enseñanza de morfosintaxis en la educación primaria debe abordarse a partir del uso, de la comprensión y de la producción de textos orales y escritos.

- Se conoce el vocabulario que dominan los estudiantes de educación primaria (léxico fundamental) y se ofrece un listado del vocabulario que se pretende enseñar en cada nivel académico (léxico meta).

- La población costarricense contará, a corto plazo, con un diccionario escolar elaborado a partir de la competencia comunicativa de los educandos y los insumos curriculares que se utilizan en la enseñanza básica.

\section{Artículos publicados en revistas especializadas}

Uno de los compromisos básicos de un centro académico es divulgar su producción intelectual. Un instrumento fundamental para lograr ese impacto en la sociedad es una publicación periódica, con el debido respaldo académico. Por eso, en este apartado se 
consideran los artículos en relación con la enseñanza y el aprendizaje del español en I y II ciclos de Educación General Básica, publicados en cuatro revistas de la Universidad de Costa Rica: Educación, Káñina, Filología y Lingüística y Actualidades en Educación.

\section{Educación: Revista de la Universidad de Costa Rica}

El objetivo de la Revista Educación de la Universidad de Costa Rica es difundir la investigación y promover reflexión académica referida al campo educativo. En esta revista solo se han publicado cuadro artículos en relación con la enseñanza de la lengua española en educación primaria.

El primero se titula "El lenguaje caracterización de sus dos formas fundamentales: el código oral y el código escrito" y fue escrito por María del Carmen Ugalde (1988), en el cual se concluye que: entre hablar y escribir hay una gran diferencia, ya que escribir es un arte que se aprende en la escuela muchos años después de que se habla (desde el nacimiento) y que debe ser aprendido y practicado constantemente en cuanto a las normas que exige el código escrito. Así, la escuela debe asumir la difícil tarea de variar la forma tradicional en que se ha enseñado la escritura, pues conviene introducir el manejo de conectores discursivos y sus significados, la organización de la información, la estructuración del párrafo, entre otros aspectos del texto escrito. Además, se resalta la necesidad de trabajar en forma paralela la escritura y la lectura.

En el segundo artículo, llamado "El maestro frente a las dificultades de la lengua oral", de María del Pilar Rodríguez (1991), reúne algunos conceptos acerca de la problemática que ofrece la adquisición del lenguaje en el niño, con el fin de que sirva como información al maestro que inicia su labor docente. Este artículo también recomienda procedimientos para detectar problemas de lenguaje en los niños y sugiere distintos tipos de actividades de recuperación y de estimulación para la audición y la articulación. Por tanto, concluye que el maestro debe tener presente que los problemas de lenguaje provocan problemas de aprendizaje, se debe proporcionar programas remediales, proporcionar un modelo verbal correcto, aceptar los impedimentos de los niños y favorecer su integración en el grupo.

En el tercer artículo “¿Práctica de palabras o de ideas? Panorama histórico de la enseñanza del léxico en Costa Rica", de Marta Rojas y Oscar Aguilar (2006) se sigue la trayectoria del tratamiento didáctico de la enseñanza del léxico en Costa Rica desde inicios del siglo XX 
con don Carlos Gagini (1897, 1902, 1904), precursor de la enseñanza del léxico como objetivo específico, también se revisan las obras de Lilia González y Manuel Clemente Quesada, quienes definen un léxico meta para enseñar ortografía a partir de la producción del estudiantado (1930); se incursiona en la década de los 40 con el aporte de Hernán Zamora Elizondo (1942), quien presenta una propuesta visionaria para la enseñanza de la lengua con objetivos comunicativos y continúa el recorrido con Claudia Cascante Rojas, Castellano (1946), obra prologada por Roberto Brenes Mesén, erudito que señala la importancia de supeditar las palabras al servicio de la comunicación. Las dos primeras décadas de la segunda mitad del siglo XX están marcadas por una propuesta comunicativa y por la consideración de las funciones sociales del lenguaje, desarrollado por Emma Gamboa, Mario Fernández Lobo e Isaac Felipe Azofeifa; también se hace referencia a los proyectos oficiales: Programa ODECA-ROCAP (1968-1978), mediante el cual se dotó de libros de texto a las escuelas públicas del país y a la Serie Hacia la Luz (1985). El último período, después de la década de los ochenta, se denomina etapa de los enfoques comunicativos o procesuales, iniciado por Víctor Ml. Sánchez Corrales, quien hace una propuesta de planificación lingüística basada en la investigación de las competencias del estudiantado y de un perfil de hablante culto. Como parte de esta misma etapa se cita la participación de Marta Rojas en los programas oficiales de 1995 y en los libros de español de la Serie Hacia el Siglo XXI. Para concluir se reseña la propuesta de Marielos Murillo Rojas, abordaje del léxico de manera integrada y partiendo de la experiencia de los niños y las niñas, y la de Marta Rojas propuesta de léxico meta para cada año de la escuela costarricense, a partir del vocabulario de los libros de la Serie Hacia el Siglo XXI y las investigaciones de Sánchez, Murillo y Rojas.

Además, en el segundo volumen del año 2006 se publicará el artículo "Esbozo de lineamientos conceptuales para la enseñanza y el aprendizaje del léxico" de Marta Rojas, documento que no se pudo consultar por estar en proceso de publicación.

Así, los artículos de la revista Educación consideran la enseñanza y el aprendizaje de la expresión oral, macrohabilidad lingüística que ha sido poco estudiada en el país. El primero, compara el código oral con el código escrito y el segundo, hace referencia a los problemas de lenguaje que pueden presentar los estudiantes y el tercero, hace un recorrido histórico por la enseñanza del léxico en la escuela costarricense. 


\section{Káñina: Revista de Artes y Letras de la Universidad de Costa Rica}

Esta publicación se dedica a la difusión, principalmente, de la literatura costarricense, pero recibe contribuciones de escritores de todas las ramas, siempre y cuando posean alto nivel académico, cultural y estético. En esta publicación periódica de la Facultad de Artes y de Letras de la UCR, se han publicado nueve artículos en relación con los procesos de enseñanza-aprendizaje del español en I y II ciclos de educación primaria.

En el artículo titulado "Comunicarse y crecer: Análisis crítico del trabajo con la lectura, la escritura, la expresión y comprensión orales en la escuela costarricense", de Marielos Murillo (2005), se analiza críticamente la enseñanza del español como lengua materna en la escuela costarricense y se proponen algunas líneas generales para fortalecer la competencia comunicativa del niño. Se concluye que urge la presencia de políticas de investigación acerca del capital lingüístico que poseen los alumnos, la modificación sustancial de los programas de estudio y la creación de procesos de formación permanente dirigidos hacia los educadores en servicio. Además, señala que debe planificarse de manera proporcional el trabajo y estudio de las cuatro destrezas y habilidades lingüísticas, de acuerdo con el nivel y el desarrollo evolutivo de los educandos. En ese sentido se considera que el educador debe ser un elemento activo y reactivo dentro del proceso de enseñanza y aprendizaje y que la escuela debe ocuparse de dar seguimiento a los procesos de apropiación de la lengua materna como medio de comunicación escolar y como objeto de estudio.

Marta Eugenia Rojas y Natalia Campos (1994), publicaron el artículo "Caracterización de los procesos de enseñanza-aprendizaje a partir del análisis de la interacción verbal en el aula costarricense", el cual corresponde a un estudio de la interacción verbal que se da en aulas de primer grado de una escuela ubicada en una comunidad urbano-marginal del país. Los objetivos de dicho estudio fueron identificar las funciones del lenguaje y analizar las relaciones entre el proceso de interacción verbal y el contexto de enseñanza-aprendizaje. En este estudio se obtuvo como principales resultados que la escuela costarricense favorece el trabajo individual en detrimento del trabajo colectivo; considera las interacciones entre los estudiantes como interrupciones; no incorpora el juego como estrategia metodológica e impone el lenguaje en un clima de normativa y rigidez, por lo que ignora o censura la variedad de lenguaje de la comunidad del niño. Por consiguiente, la escuela es un agente de socialización, cuyo estilo de enseñanza-aprendizaje revela una transmisión de normas sociales de dependencia y de sumisión en relación con la autoridad, pues el énfasis en el 
estilo de control y guía directiva (directivo) conduce a la obediencia, a la pasividad y a la dependencia.

Murillo y Sánchez (2002) en "Crecimiento del léxico básico de los escolares costarricenses: código oral", artículo derivado de la investigación Léxico básico escolar costarricense ofrecen datos basados en la producción textual discursiva de escolares. Entre sus hallazgos generales encontraron que las tres categorías gramaticales con mayor representación son, respectivamente, los sustantivos, los verbos y los adjetivos.

En el artículo titulado "En torno al nombre adjetivo en el discurso oral de los niños escolares costarricenses: lineamientos para su enseñanza”, de Marielos Murillo (2002), se analizan los adjetivos presentes en el LEBAESCO -Léxico básico escolar costarricense-, a partir de tres clases léxico-sintácticas de adjetivos. En este artículo se concluye que el adjetivo confiere precisión a los textos y enriquece la prosa, por lo que es imperativo que se le dedique un espacio mayor en la enseñanza de la lengua como una categoría gramatical que enriquece el léxico.

En el artículo "La enseñanza del vocabulario en la Educación General Básica: propuesta metodológica”, de Marielos Murillo (1999), se resalta la importancia del componente léxico en la escuela y se orienta la enseñanza del vocabulario en forma articulada, con el fin de avanzar paralelamente con la realidad del educando y con el resto de las temáticas por estudiar. Con el objetivo de desarrollar la competencia léxica en los estudiantes, la autora hace una propuesta metodológica en la que plantea el uso del diccionario como un libro que permite adquirir conciencia del uso responsable de la lengua y no solamente como instrumento de consulta de significados y ortografía, que debe elegirse y emplearse adecuadamente según las necesidades y las etapas de desarrollo de los usuarios. También propone aprender vocabulario mediante la ampliación de campos semánticos -estructuras de vocabulario que informan la visión de mundo de los hablantes-; del análisis de rasgos semánticos -conocimientos previos de los alumnos y forma de almacenamiento y recuperación- y de la elaboración de esquemas y organigramas.

El artículo "Vocabulario cacográfico. Pautas para la enseñanza de la ortografía en la escuela primaria costarricense", de Marielos Murillo (2006), tiene como propósito identificar los comportamientos y patrones generales de la ortografía de los niños escolares costarricenses. 
En este artículo se concluyó que los dos problemas mayores en ortografía son la marcación gráfica del acento, según las reglas establecidas; y la escritura de los fonemas /b/, /s/, /y/, /r/ y la hache; los escolares no saben cuándo se marca el acento y cuándo no; las palabras también, así, más, después y los verbos haber, estar, hacer y tener son más difíciles que otros vocablos, pese a su elevado índice de uso; los homófonos también son conflictivos por lo que deben estudiarse en pares, teniendo en cuenta su función dentro del discurso; muchos sustantivos y adjetivos de uso común se escriben sin ortografía, probablemente por el uso de métodos de enseñanza-aprendizaje poco efectivos.

El artículo "Diaintegración, contrastividad y vocabulario de los escolares costarricenses”, de Víctor Manuel Sánchez y Marielos Murillo (2004), se propone identificar el vocabulario contrastivo y casos de transferencia léxica de algunas lenguas distintas al español o variedades internas a este, incorporados en la competencia comunicativa de los niños escolares de Costa Rica, como muestra de un proceso social y nivelador que ha permitido configurar usos lingüísticos nacionales y, con ello, la construcción de una identidad cultural en correlación con nuestra correspondiente variedad de lengua, manifiesta en el vocabulario. En este se concluye que: 1) existe la presencia de un número significativo de indigenismos propios de grupos aborígenes costarricenses (nahuatlismos, arahuaco-caribismos y quechuismos), 2) el uso de marinerismos y de otro número importante de palabras desusadas en el español peninsular, pero de uso actual en el costarricense, corroboran una vez más que el calificativo de arcaísmos léxicos debe aplicarse con cautela, pues lo que es un arcaísmo en una región no lo es en otra del mismo mundo hispánico, que comparte un idioma, 3) se encuentran tecnicismos que han pasado a la lengua común en virtud de los acontecimientos sociohistóricos que vive la sociedad actual, por ejemplo clonación, internet, etc., 4) por último, los anglicismos son los extranjerismos más numerosos en el vocabulario de los escolares; algunos ya han adquirido la categoría de préstamos estables, pues son de competencia y uso de los escolares.

Por otra parte, el artículo titulado "Análisis sintáctico de textos orales y escritos de niños escolares costarricenses. Aspectos básicos para la programación del aprendizaje lingüístico escolar", de Marielos Murillo y Víctor Manuel Sánchez (2007), expone los resultados del proyecto de investigación "Análisis sintáctico de textos orales y escritos de niños preescolares y escolares costarricenses. Fundamentos para la programación del aprendizaje lingüístico escolar". En este se concluyó que los niños poseen mayor madurez sintáctica en 
los textos escritos, probablemente porque la escuela dedica más tiempo al código escrito que al oral, cuando ambas deben ocupar un lugar privilegiado en los programas de lengua materna, fundamentalmente en los primeros niveles de enseñanza. Además, el sexo es una variable que no determina la madurez sintáctica en el código oral ni en el escrito, pero la variable tipo de escuela sí marca diferencias entre grupos, estableciendo brechas entre los escolares que asisten a escuelas rurales, respecto de los de instituciones urbanas y privadas.

Y el artículo "La lectura según opiniones de niños escolares costarricenses”, de Marielos Murillo (1998), es un estudio que determina el estatus que posee la lectura en los escolares que finalizan la educación general básica, pues ellos son el producto de un sistema educativo que contempla, dentro de sus objetivos, la promoción de la lectura. En este se constató que los estudiantes sienten gusto por la lectura, pero les disgusta la forma en que los docentes dan sus lecciones; rechazan las actividades escolares de lectura y escritura; dedican más tiempo libre a practicar deporte, escuchar música y ver televisión; prefieren leer las tiras cómicas y los deportes en los periódicos; visitan poco la biblioteca; no encuentran libros en la escuela que respondan a sus intereses; y en el hogar observan poco estímulo hacia el hábito de la lectura.

En resumen, cinco (55.55\%) de los artículos se refieren al vocabulario de los escolares costarricenses (uno especifica el uso de adjetivos); mientras que solamente uno de estos $(11.11 \%)$ se refiere a la interacción verbal dentro del aula; otro artículo trata sobre la madurez sintáctica de los niños; otro, analiza la enseñanza de las cuatro macrohabilidades lingüísticas; y el último, analiza la opinión de los estudiantes con respecto a la lectura.

De estos se puede inferir que la enseñanza de la lengua en la escuela costarricense es academicista, pues se centra en conocimientos y no en competencias; favorece la formación de sujetos pasivos que deben limitarse a escuchar y no a interactuar con el profesor; da énfasis al estudio del código escrito y no el proceso de escritura; y no promueve la lectura. Por consiguiente, se necesita desarrollar en los estudiantes, en forma integral, las cuatro habilidades comunicativas -lectura, escritura-habla y escucha-, mediante técnicas innovadoras, a la luz de un nuevo enfoque de enseñanza y aprendizaje de la lengua. 


\section{Revista de Filología y Lingüística de la Universidad de Costa Rica}

El objetivo de esta revista es permitir la publicación de artículos y trabajos de investigación sobre temas relevantes para la comunidad local, nacional e internacional. En esta se han publicado dos artículos en relación con la enseñanza del español en la escuela primaria.

En el artículo "La lengua española en la educación costarricense, redefinición", Víctor Manuel Sánchez (1989) expone un ensayo sobre la evolución que ha tenido en América el concepto de lengua española y lo redefine en la educación costarricense. De acuerdo con él, enseñar español en la educación costarricense ha de ser enseñar las distintas variedades del español que hay en el país, de modo que el educando pueda desenvolverse adecuadamente, según la situación comunicativa. Para constatar la variedad de registros lingüísticos en el español de Costa Rica es necesario codificar esas variedades en los distintos niveles: fonética y fonología, morfosintaxis, léxico y semántica. Además, es muy importante el trabajo de investigación del registro culto sobre la base de metodologías y marcos teóricos rigurosos, tanto para el análisis como para la obtención de córpora representativos; eliminar prejuicios de educadores y alumnos para legitimar la norma culta nacional; y despertar actitudes de lealtad y de orgullo hacia las variedades lingüísticas costarricenses, pues el uso lingüístico nos distingue en el conjunto de pueblos centroamericanos, hispanoamericanos y peninsulares.

Y el artículo de revista titulado "Crecimiento de la disponibilidad léxica: niños de preescolar y primer ciclo de la educación básica costarricense”, de Marielos Murillo (1999), determina el número de términos diferentes usados en cada nivel y su relación con las variables sociolingüísticas: sexo, nivel sociocultural y tipo de escuela (pública urbana, pública rural y marginal). En este estudio se concluyó que la escolaridad incrementa la disponibilidad léxica, pues el mayor crecimiento de vocabulario se da de kinder a primer grado mientras que de primero a segundo y de segundo a tercero el patrón de crecimiento avanza más lentamente. Por último, se sostiene que la enseñanza de nombres específicos es una tarea reciente y compete a la escuela, que debe proporcionar las herramientas necesarias para que el niño sea un hablante eficiente.

Por consiguiente, uno de los artículos de la revista de Filología y Lingüística de la UCR conceptualiza la lengua española en el país; y el otro, expone el crecimiento del vocabulario básico en los escolares según sus niveles académicos. 


\section{Revista Electrónica Actualidades Educativas del Instituto de Investigaciones en Educación (INIE)}

El objetivo de esta revista es permitir la publicación de artículos y trabajos de investigación en el campo educativo. Por tanto, presenta siete artículos en relación con la enseñanza y el aprendizaje de la lengua española en educación primaria.

Para empezar, el artículo "La apropiación de la lengua escrita: un proceso constructivo, interactivo y de producción cultural", de Ana Lupita Chávez (2001), hace referencia a investigaciones y a teorías que conciben la apropiación de la lengua escrita por parte del sujeto como un proceso constructivo, interactivo y de producción cultural, con el propósito de invitar a los educadores a reflexionar sobre la pertinencia de las prácticas pedagógicas que se desarrollan en los centros educativos del país.

El artículo titulado "Formación docente en el área de lengua en las universidades estatales de Costa Rica", de Marielos Murillo (2007), tiene como propósito revisar la pertinencia de la formación en el área de lengua, en los programas de formación de maestros de primaria en las universidades estatales. En este se concluye que las universidades estatales desarrollan tres aspectos fundamentales en el área de español: literatura infantil, didáctica de la lectoescritura y didáctica de la lengua española; siendo esta última la que recibe un tratamiento más tradicional y desligado de las características del objeto de estudio. Dada esta situación se recomienda que en los programas de formación docente se conceptualice la lengua como área instrumental para la socialización y la adquisición de nuevos conocimientos, se destine un espacio curricular más amplio al estudio integral de cada una de las macrohabilidades del lenguaje y las microhabilidades asociadas a estas y se desarrollen habilidades en los docentes para estudiar la secuencialidad y profundidad de los contenidos curriculares del área de lengua, a la luz de las características evolutivas de los educandos.

El artículo "Las estrategias didácticas en la formación de docentes de educación primaria", de Wilfredo Gonzaga (2005), da a conocer los resultados de un estudio sobre el proceso didáctico y la incorporación de estrategias innovadoras en los procesos de enseñanza y aprendizaje que se llevan a cabo en el desarrollo de los cursos que conforman el área pedagógica del plan de estudio, para la formación de docentes en educación primaria, en la Sede de Occidente de la Universidad de Costa Rica. Dicha investigación demostró que el 
plan de estudio considera como referencia pedagógica un enfoque humanista, mientras que los programas de los cursos y la práctica docente no logran desligarse de la didáctica tradicional, con tendencia a incorporar aspectos del constructivismo y de la pedagogía crítica, pero sin la incorporación de estrategias innovadoras de manera significativa.

El artículo "Léxico meta para una propuesta metodológica para el desarrollo de la madurez léxica en el primer ciclo de la Educación General Básica”, de Marta Rojas (2005), corresponde a la publicación de su trabajo final de graduación titulado "Léxico meta para la escuela costarricense: esbozo para una propuesta metodológica para el desarrollo de la madurez léxica", ya expuesto. De este se concluyó que el léxico meta de los escolares está formado por 1816 palabras, que deberían enseñarse en primer ciclo de la Educación General Básica.

El artículo "Léxico meta para la escuela costarricense”, de Marta Rojas (2006),_describe los procesos seguidos para la definición de un léxico meta por enseñar en cada año de I y II ciclos de la educación primaria costarricense. Por tanto, su propósito es compartir los procesos seguidos en el estudio Léxico meta para la escuela costarricense: esbozo para una propuesta metodológica para el desarrollo de la madurez léxica, llevado a cabo en el INIE y como trabajo de graduación de la Maestría en Lingüística de la Universidad de Costa Rica (por lo que ya fue comentada en los trabajos finales de graduación de la UCR).

En el artículo "El XSLT como herramienta para el diseño de sistemas lexicográficos informatizados: aplicación a adjetivos de un léxico meta", de Marta Rojas y Antonio Leoni de León (2005), se expone el procedimiento seguido para la clasificación y análisis de los adjetivos de un léxico meta propuesto para el II ciclo de la Educación General Básica. En este se concluyó que el XSLT es una herramienta de gran valor para el diseño de sistemas lexicográficos informatizados pues, entre otras ventajas, favorece la manipulación de corpus amplios, por parte de quienes investigan y planifican la enseñanza y la incorporación del léxico a la competencia comunicativa de la niñez costarricense.

En el artículo "La lectura en la escuela costarricense. Algunas reflexiones" de Marielos Murillo (2005) se sostiene que el objetivo fundamental de dicha investigación fue analizar la concepción de lectura que subyace en los programas de español de I y II ciclos del Ministerio de Educación Pública (MEP) y su adecuación al desarrollo del niño en cada una de las etapas de 
la escolaridad. Así, se concluye que: La formación teórico-práctica del docente debe focalizar en su trabajo, en forma integral y consciente, las habilidades lingüísticas, en el presente caso la lectura. Las universidades, como centros difusores de cultura, deben comprometerse con programas de animación a la lectura, círculos de lectura y discusión de problemas que atañan a las didácticas del contenido, mediante estrategias de educación continua y de apoyo directo al Ministerio de Educación. Y finalmente, la organización del trabajo escolar debe fomentar las capacidades de los alumnos y sus responsabilidades.

El artículo titulado "El verbo en la escritura cacográfica de los niños escolares costarricenses. Algunos lineamientos para su enseñanza", de Marielos Murillo (2005), si bien se publica en la Revista Campo Abierto de la Universidad de Extremadura, se incluye en vista de que la temática tratada forma parte de la línea de investigación seguida en este artículo y los datos que se analizan corresponden a una muestra de producciones textuales de escolares costarricenses. Ahora bien, este presenta artículo expone como principales hallazgos la determinación de las palabras con mayor variación en la escritura de los niños (verbos, adverbios, sustantivos, adjetivos, preposiciones, pronombres y conjunciones, respectivamente) y la reincidencia de errores ortográficos grafémicos o de acentuación por parte de los escolares al usar formas verbales de la primera, segunda y tercera conjugación en el discurso. Además, ofrece algunos lineamientos para la enseñanza de la escritura de las formas verbales en la escuela costarricense, lo cual constituye un aporte muy valioso para la enseñanza y el aprendizaje de la lengua española en la educación primaria costarricense.

Además, por la relación con el tema del presente documento se incluye el artículo " $\mathrm{La}$ enseñanza de la lengua española en la educación primaria costarricense. Aportes de la investigación lingüística y educativa", de Marielos Murillo, por aparecer en RILI -Revista de Internacional de Lingüística Iberoamericana, Alemania-, en el cual se señala que la lengua española en la escuela costarricense, según muestran los resultados de pruebas nacionales, diagnósticos que valoran el rendimiento académico en la asignatura de español y las investigaciones realizadas en las universidades estatales de Costa Rica, evidencia una crisis en el dominio de las competencias comunicativas básicas de los escolares. Esas competencias son fundamentales para vivir en sociedad y, por tanto, básicas a nivel escolar, pues constituyen el soporte de los procesos de enseñanza y aprendizaje. 
Se considera que Costa Rica vive todavía un período de transición en el que el tradicional enfoque gramatical continúa fuertemente asentado en la estructura escolar, tal y como lo evidencian los tres últimos programas estatales de español (1995, 2001 y 2005) para la educación primaria (cf. Murillo 2007).

Por otra parte, los docentes continúan apegados a prácticas tradicionales en la enseñanza de la lectura y la escritura y conceptualizan la lengua materna como asignatura y no como área instrumental para la adquisición de conocimientos y la socialización.

En vista de lo señalado, el artículo en comentario se ocupa de indagar cuáles son los aportes, a nivel de investigación, que han realizado las universidades estatales de Costa Rica (Universidad de Costa Rica -UCR-, Universidad Nacional de Costa Rica -UNA-y Universidad Estatal a Distancia -UNED-), desde 1990 al año 2007, respecto de la enseñanza y aprendizaje de la lengua española en la educación primaria. Para tal efecto se revisaron 41 trabajos finales de graduación, 13 proyectos de investigación y 21 artículos publicados en revistas especializadas, para un total de 75 trabajos publicados.

De las investigaciones consultadas se consideran los hallazgos relacionados con: el rendimiento académico en el área de español, las metodologías específicas para la enseñanza de la lengua, las áreas lingüísticas objeto de investigación, los trabajos orientados hacia la mediación pedagógica, la pertinencia de los planes de estudio estatales, los estudios sobre el capital lingüístico de los alumnos y la formación de los docentes en el área de lengua.

En resumen, el primer artículo de la revista Actualidades en Educación expone la apropiación de la lengua escrita por parte del sujeto como un proceso constructivo, interactivo y de producción cultural. El segundo analiza la formación de los docentes en el área de lengua. El tercero analiza las estrategias didácticas en la formación de docentes de educación primaria. El cuarto ofrece algunos lineamientos para la enseñanza de la escritura de las formas verbales en la escuela costarricense. El quinto presenta una propuesta del léxico básico por enseñar en el primer ciclo de educación primaria. El sexto evalúa el programa XSLT como una herramienta para el diseño de sistemas lexicográficos informatizados. Por último, el sétimo artículo invita a reflexionar sobre la lectura en la educación primaria costarricense. 
Por consiguiente, a manera de síntesis se puede decir que en la Universidad de Costa Rica, se han publicado veintidós artículos de revista en relación con la enseñanza y el aprendizaje de la lengua española en educación primaria.

De estos, seis (30\%) conciernen a la enseñanza de la lengua escrita y el desarrollo de sus microhabilidades; siete (25\%) tratan sobre el léxico de los escolares; dos (10\%) reflexionan sobre la enseñanza de la lectura; dos (10\%) atañen a la formación de docentes en el área de lengua y los cinco artículos restantes se ocupan en forma individual de: el tratamiento integral de las cuatro áreas lingüísticas, la comparación del código oral con el escrito, la señalización de dificultades en el lenguaje oral, el estudio de la interacción verbal y la evaluación del programa XSLT para sistemas lexicográficos ( $5 \%$ cada uno).

Consecuentemente, dichos artículos ofrecen los siguientes hallazgos generales:

- La formación de los docentes en las universidades estatales, área de lengua, se centra en el abordaje metodológico de la literatura infantil, la didáctica de la lectoescritura y la didáctica de la lengua; con un enfoque academicista basado en contenidos y no en el desarrollo de competencias de los alumnos. Sumado a eso los educadores carecen de formación suficiente para atender adecuadamente el desarrollo de la competencia comunicativa en los escolares.

- La escuela debe impulsar la madurez léxica de los escolares, a partir del estudio del vocabulario meta que se propone para cada nivel educativo.

- Es crucial que la escuela adopte un nuevo concepto de lectura como proceso interactivo, en el que el lector es considerado un sujeto activo que utiliza conocimientos de tipo muy variado para obtener información del texto y construir significados, de acuerdo con sus propios esquemas conceptuales. También es importante que desarrolle estrategias de promoción y animación a la lectura.

- La enseñanza y el aprendizaje de la lengua española costarricense es tradicional. Por tanto, resulta necesario que se implemente el enfoque comunicativo de la lengua para desarrollar integralmente las cuatro destrezas o macrohabilidades lingüísticas: lectura, escritura, habla y escucha.

Además, la mitad de los artículos en mención se fundamentan en revisiones bibliográficas 0 en opiniones de informantes y la otra mitad (50\%) se basan en pruebas de ejecución como la elaboración de textos escritos u orales por parte de los escolares. 
Por último, cabe señalar que el 55\% de los artículos sí exponen lineamientos metodológicos para el mejoramiento de la enseñanza y el aprendizaje de la lengua española en la educación primaria costarricense.

\section{Conclusiones}

Desde 1990 hasta la fecha, en la Universidad de Costa Rica se han divulgado cincuenta y tres trabajos de investigación en relación con la enseñanza y el aprendizaje de la lengua española en educación primaria.

La mayoría de estos trabajos (64.71\%) trata sobre el desarrollo de microhabilidades lingüísticas por parte de los escolares como son la redacción, el vocabulario básico, la ortografía, el uso de puntuación, la sintaxis y la comprensión de lectura; correspondientes a las áreas de lectura y escritura. El $13.73 \%$ de las investigaciones presenta el rendimiento académico de los estudiantes de primaria en la asignatura de español. El $9.80 \%$ trata sobre la formación de docentes en el área de lengua. Un 5.88\% considera el código oral. El 3.92\% de las investigaciones atañe, directamente, a la metodología y a la didáctica que emplean los docentes de I y II ciclo de Educación General Básica en la asignatura de español. Y el restante $1.96 \%$ (una publicación) se refiere al tratamiento integral que debería darse a las cuatro áreas lingüísticas.

Los resultados obtenidos en tales trabajos evidencian una crisis en la enseñanza y el aprendizaje de la lengua española en la educación primaria costarricense, pues, los docentes no poseen los conocimientos ni las destrezas necesarios para abordar la asignatura de español, por lo que emplean métodos y estrategias tradicionales en la enseñanza de esta y, en consecuencia, los niños y niñas de educación primaria manifiestan debilidad en el manejo de las macrohabilidades lingüísticas.

Cabe señalar también que treinta y seis (68.63\%) de estos trabajos de investigación se basan en pruebas de ejecución realizadas por los informantes, tales como producciones textuales escritas $u$ orales. Sin embargo, los dieciséis trabajos restantes solamente se fundamentan en revisiones bibliográficas o en opiniones recolectadas mediante entrevistas, cuestionarios o grupos focales. Por tanto, se evidencia la necesidad de investigar el capital lingüístico de los educandos y la práctica pedagógica de los educadores, con el objetivo de 
sustentar los procesos de planificación lingüística en la competencia de los sujetos de la educación y en la actuación de los diferentes elementos que entran en juego en el proceso educativo.

Además, es preocupante que treinta (58.82\%) de estos trabajos de investigación son simples diagnósticos de la realidad educativa que se limitan a señalar las fallas en los procesos educativos sin ofrecer soluciones pertinentes, en contraste, solamente veintidós $(41.18 \%)$ de los trabajos comentados exponen lineamientos 0 estrategias metodológicas para el desarrollo de las competencias lingüísticas de los escolares y el mejoramiento de la enseñanza y el aprendizaje de la lengua española en la educación primaria costarricense. Por lo tanto, es primordial que la Universidad de Costa Rica asuma la investigación sobre temas relevantes para la educación nacional e incorpore el planteamiento de propuestas que inviten a revertir la lamentable situación que se vive en las aulas costarricenses.

De esta manera, se puede concluir que las principales líneas de investigación han sido diagnósticos sobre la acción pedagógica de los educadores; sobre el rendimiento académico de los estudiantes en la asignatura de español en general o sobre el dominio de microhabilidades lingüísticas como son la comprensión de lectura, la producción textual redacción-, el vocabulario básico, el uso de puntuación y la ortografía, correspondientes a las áreas de lectura y escritura; la expresión oral es un área poco explorada en el país y la escucha es todavía un área inexplorada. En este sentido, la investigación refleja un abordaje desintegrado de la lengua española que, lamentablemente, no se conceptualiza como proceso sino como producto.

Asimismo, cabe recalcar la realización de varias investigaciones basadas únicamente en trabajos de opinión y que carecen de propuestas funcionales para revertir la situación señalada en la enseñanza y el aprendizaje de la lengua española en la escuela costarricense.

Por consiguiente, en la Universidad de Costa Rica resulta necesario incrementar la investigación de los procesos de enseñanza y aprendizaje de las cuatro macrohabilidades lingüísticas -lectura, escritura, habla y escucha-, a partir de trabajos prácticos ejecutados por los sujetos en estudio y con el objetivo de realizar propuestas para mejorar la realidad educativa que se describe. También es fundamental ofrecer una formación inicial más 
completa a los docentes y comprometerlos en programas de actualización donde se incorporen sustentos epistemológicos, teóricos y metodológicos para una mejor formación en relación con la enseñanza de la lengua.

\section{Referencias}

Aguilar, Hazel, Barboza, Diana, Barth, Eugenie, Montero, Anabelle, Peña, Yessenia y Zúñiga, Olany. (2007). La implementación de la filosofía del lenguaje integral para el desarrollo de la lectoescritura en un grupo de la Educación General Básica. Tesis de Licenciatura en Educación Primaria, Universidad de Costa Rica, San José, C.R.

Aguilar, M. (2000). El lenguaje integral: Una experiencia con niños en riesgo social. Tesis de Licenciatura, Universidad de Costa Rica, San José, C.R.

Barquero, Ana Catalina, Calderón, Lucrecia, Díaz, Teresita, Porras, Sylvia y Vega, Kattia. (2004). La animación y promoción de lectura por medio de la caja viajera como un recurso de comunicación entre la escuela y el hogar. Tesis de Licenciatura en Educación Primaria, Universidad de Costa Rica, San José, C.R.

Benavides, Jennory, Brenes, María Lorena, Coto, Maritza, Espinoza, Clara, Ibarra, Shirley y Rojas, Norma. (1996). Competencia en la comprensión de lectura de escolares que finalizan la Educación General Básica. Tesis de Licenciatura en Educación Primaria, Universidad de Costa Rica, San José, C.R.

Bogantes, A. (1989). Dominio de contenidos programáticos mínimos de ciencias, español, estudios sociales y actitud hacia la docencia de los aspirantes que solicitan ingresar a los planes de formación de los docentes, mediante el convenio Ministerio de Educación Pública-Universidades. Tesis de licenciatura, Universidad Estatal a Distancia, de Costa Rica, San José, C.R.

Bonilla, Martiza, Prendas, Ligia, Monestel, Patricia y Porras. Martiza. (1994). Lectura creadora: una propuesta para incrementar las habilidades de comprensión lectora y expresión escrita. Tesis de Licenciatura, Universidad de Costa Rica, San José, C.R.

Carballo, Melina y Mairena, Nuria. (1997). Evaluación curricular del plan de bachillerato en educación primaria de la Sede de Guanacaste. Tesis de Maestría con énfasis en Administración Educativa, Universidad de Costa Rica, Guanacaste, C.R.

Campos, Isabel, Ledesma Vera, Rivera, Sheila y Villalobos, Guiselle. (1998). Análisis de algunos factores que incidieron en el fracaso escolar de los alumnos reprobados en cuarto grado de la Dirección Regional de Educación de San Ramón en 1996. Tesis de Licenciatura, Universidad de Costa Rica, Alajuela, C.R.

Carvajal, L. Fuentes, C. y Miranda, M. (1997). El uso de formas en la enseñanza de las sílabas consonánticas en segundo grado de Educación General Básica en el cantón de Orotina. Tesis de Licenciatura, Universidad Estatal a Distancia, Alajuela, C.R. 
Castrillo, C. Morales, M. Ocampo, F. Pacheco, E. y Wong, C. (2007). El empleo de la puntuación en la educación primaria costarricense. Tesis de Licenciatura, Universidad de Costa Rica, San José, C.R.

Chacón, Jenny. (2005). La expresión escrita: un medio para explorar la vida cotidiana de los niños y las niñas de tercer grado. Tesis de Licenciatura en Educación Primaria, Universidad de Costa Rica, San José, C.R.

Chávez, Lupita. (2001). La apropiación de la lengua escrita: un proceso constructivo, interactivo y de producción cultural. Revista Electrónica Actualidades Investigativas en Educación, 1 (1). Recuperado el 25 de julio 2007, de http://revista.inie.ucr.ac.cr/articulos/1-2001/archivos/apropiacion.pdf

Cortés, D. González, R. Monge, S. Quesada, S. y Washington, P. (1997). Crecimiento de la disponibilidad léxica en estudiantes de la Zona de Turrialba de nivel preescolar y primer ciclo de la Educación General Básica. Tesis de Licenciatura, Universidad de Costa Rica, Cartago, C.R.

Esquivel, Juan Manuel. (1990). Medición de la calidad de la enseñanza. San José, Costa Rica: IIMEC.

Esquivel, Juan Manuel. (1994). Medición de la calidad de la educación UNESCO-OREALIIMEC. San José, Costa Rica: IIMEC.

Esquivel, Juan Manuel. (1995). Informe ejecutivo de la administración de las pruebas de diagnóstico de español y matemáticas abril-mayo, 1995. San José: IIMEC.

Esquivel, Juan Manuel. (1996). Informe político del desarrollo, validación y aplicación de las pruebas de diagnóstico. San José: IIMEC.

Fajardo, Yalena, López, Alexandra, Montoya, Carmen, Romero, Nancy, Salas, Yesenia y Zumbado, Carmen. (2006). La lectura interactiva como medio para incrementar habilidades de comprensión de lectura y expresión escrita. Tesis de Licenciatura, Universidad de Costa Rica, San José, C.R.

Fallas, Melba. (2005). Lineamientos metodológicos para la integración del tema transversal-vivencia de los derechos humanos- para la democracia y la paz y la enseñanza del español en quinto año de la EGB. Tesis de Maestría en Planificación Curricular, Universidad de Costa Rica, San José, C.R.

Fuentes, Diana, De la O, Orlando, Ortega, Sonia y Zamora, Lizbeth. (1998). Ventajas y desventajas del trabajo por áreas o materias de estudio, según opiniones de niños, docentes, padres de familia y autoridades, en las escuelas privadas e instituciones técnicas públicas del circuito 02, Liberia. Tesis de Licenciatura en Educación Primaria, Universidad de Costa Rica, Guanacaste, C.R.

Gonzaga, Wilfredo. (2005, 30 de junio). Las estrategias didácticas en la formación de docentes de educación primaria. Revista Actualidades Investigativas en Educación, Universidad de Costa Rica, Vol. 5 (1). Recuperado el 25 de julio 2007 de http://revista.inie.ucr.ac.cr/articulos/1-2005/articulos/estrategias.pdf 
López, Olimpia. (1990). Análisis contextual de los planes de estudio para la formación de docentes de enseñanza primaria en la Facultad de Educación de la Universidad de Costa Rica. Tesis de maestría en Sociología, Universidad de Costa Rica, San José, C.R.

Lukas, Susana y Granados, Xinia. (2004). Aplicación de técnicas didácticas y su incidencia en el aprendizaje de la redacción de los niños y niñas de tercer grado de las escuelas Atilia Mata Freses y Santa Eduviges del cantón Central de Limón. Tesis de Licenciatura en Educación Primaria, Universidad de Costa Rica, Limón, C.R.

Meza, Ana Cristina. (1994). Estudio del rendimiento académico de los alumnos de sexto año de Educación General Básica de la dirección regional de San José. Tesis de Maestría en Educación, Universidad de Costa Rica, San José, C.R.

Murillo, M. (1998). La lectura según opinión de niños escolares costarricenses. Káñina: Revista Artes y Letras, Universidad de Costa Rica. Vol. XXII (1), 107-115.

Murillo, Marielos. (1999). La enseñanza del vocabulario en la Educación General Básica: Propuesta metodológica. Káñina: Revista Artes y Letras, XXIII (2), 155-162. Universidad de Costa Rica.

Murillo, Marielos. (1999). Crecimiento de la disponibilidad léxica: niños de preescolar y primer ciclo de la educación básica costarricense. Revista de Filología y Lingüística de la Universidad de Costa Rica, XXV (2), 187-203.

Murillo, Marielos. (2003). En torno al nombre adjetivo en el discurso oral de los niños escolares costarricenses: lineamientos para su enseñanza. Káñina: Revista Artes y Letras, XXVII (1), 171-181. Universidad de Costa Rica.

Murillo, Marielos. (2004). Estudio sobre el lenguaje de los escolares costarricense: el léxico básico, la ortografía y sus características. Tesis Doctoral, Universidad de Extremadura, España.

Murillo, Marielos. (2005). Comunicarse y crecer: Análisis crítico del trabajo con la lectura, la escritura, la expresión y comprensión orales en la escuela costarricense. Káñina: Revista Artes y Letras, XXIX, (1). Universidad de Costa Rica

Murillo, Marielos. (2005). El verbo en la escritura cacográfica de los niños escolares costarricenses. Algunos lineamientos para su enseñanza. Revista Campo Abierto. (27), 23-39.

Murillo, Marielos. (2006). Vocabulario cacográfico. Pautas para la enseñanza de la ortografía en la escuela primaria costarricense. Káñina: Revista Artes y Letras, XXX (1). Universidad de Costa Rica.

Murillo, Marielos. (2007, 30 de agosto). Formación docente en el área de lengua en las universidades estatales de Costa Rica. Revista Actualidades Investigativas en Educación, 7 (2), 1-34. Recuperado el 30 de agosto 2007 de http://revista.inie.ucr.ac.cr/articulos/2-2007/archivos/lengua.pdf 
Murillo, Marielos. (2005, 15 de diciembre). La lectura en la escuela costarricense. Algunas reflexiones. Actualidades en educación: Revista Electrónica Actualidades en Educación, 5 (2) julio-diciembre. Recuperado el 25 de julio 2007 de http://revista.inie.ucr.ac.cr/articulos/2-2005/archivos/lectura.pdf

Murillo, Marielos. (en prensa). La enseñanza de la lengua española en la educación primaria costarricense. Aportes de la investigación lingüística y educativa. RILI Revista de Internacional de Lingüística Iberoaméricana, Alemania.

Murillo, Marielos y Sánchez, Víctor. (2002). Crecimiento del léxico básico de los escolares costarricenses: código oral. Káñina: Revista Artes y Letras, XXVI (2), 97-108. Universidad de Costa Rica.

Murillo, Marielos y Sánchez, Víctor. (2007). Análisis sintáctico de textos orales y escritos de niños escolares costarricenses. Aspectos básicos para la programación del aprendizaje lingüístico escolar. Káñina: Revista Artes y Letras, XXXI (1). Universidad de Costa Rica.

Murillo, Marielos y Sánchez, Víctor. (2007). Del léxico fundamental de los niños preescolares y escolares costarricenses al léxico meta y su enseñanza (Proyecto de investigación del Instituto de Investigaciones en Lingüística). San José: Universidad de Costa Rica.

Quirós, Guiselle. (2000). La aventura de leer y escribir: guía metodológica para promover la lectura y la producción textual escrita en primer ciclo de educación primaria. Tesis de Maestría en Planificación Curricular, Universidad de Costa Rica, San José, C.R.

Rodríguez, Pilar. (1991). El maestro frente a las dificultades de la lengua oral. Educación: Revista de la Universidad de Costa Rica, XV (2), 49-57.

Rojas, Marta. (2005, febrero). Léxico meta para una propuesta metodológica para el desarrollo de la madurez léxica en el primer ciclo de la Educación General Básica. Ponencia presentada en el Congreso Internacional de Investigación Educativa del $1^{\circ}$ a 4 de febrero. Instituto de Investigación en Educación, Universidad de Costa Rica. Disponible en http://www.inie.ucr.ac.cr/congreso/memoria/archivos/ponencias/martarojas.pdf

Rojas, Marta. (2006). Léxico meta para la escuela costarricense: esbozo de una propuesta metodológica para el desarrollo de la madurez léxica. Tesis de Maestría, Universidad de Costa Rica, San José, C.R.

Rojas, Marta. (2006). El léxico meta para la escuela costarricense. Revista Actualidades Investigativas en Educación, 6 (3). Recuperado el 25 de julio 2007 de http://revista.inie.ucr.ac.cr/articulos/3-2006/archivos/lexico.pdf

Rojas, Marta. (2007). Propuesta metodológica para el desarrollo de la madurez léxica: la elaboración de material didáctico para la incorporación del léxico meta (Proyecto de investigación del Instituto de Investigaciones en Educación -INIE). San José: Universidad de Costa Rica. 
Rojas, Marta, Aguilar, Oscar. (2006). ¿Práctica de palabras o de ideas? Panorama histórico de la enseñanza del léxico en Costa Rica. Educación: Revista de la Universidad de Costa Rica, 30 (1), 103-126.

Rojas, Marta. (2006). Esbozo de lineamientos conceptuales para la enseñanza y el aprendizaje del léxico. Educación: Revista de la Universidad de Costa Rica, 30 (2).

Rojas, Marta y Campos, Natalia. (1994). Caracterización de los procesos de enseñanzaaprendizaje a partir del análisis de la interacción verbal en el aula costarricense. Káñina: revista de artes y letras de la Universidad de Costa Rica, XVIII (2), 141157.

Rojas, Marta y Sánchez, Víctor. (2003). Desarrollo de la madurez léxica: propuesta metodológica. San José: IIMEC.

Rojas, M. y Sánchez, V. (2005). Léxico básico escolar costarricense: estudio de frecuencias. San José: INIE.

Rojas, Marta y Leoni, Antonio. (2005). EI XSLT como herramienta para el diseño de sistemas lexicográficos informatizados: aplicación a adjetivos de un léxico meta. Revista Electrónica Actualidades Investigativas en Educación, 5 (Extraordinario). Recuperado el 25 de julio 2007 de http://revista.inie.ucr.ac.cr/articulos/extra2005/archivos/lexico.pdf

Rubio, Carlos. (2007). El rincón de cuentos, un lugar para libros, sueños y esperanzas. Proyecto de acción social. San José: MEP-UCR.

Sánchez, Víctor. (1989). La lengua española en la educación costarricense, redefinición. Revista de filología y lingüística de la Universidad de Costa Rica, XV (2), 155-161.

Sánchez, Víctor y Murillo, Marielos. (2006). Análisis sintáctico de textos orales y escritos de niños preescolares y escolares costarricenses. Fundamento para la programación del aprendizaje lingüístico escolar. San José: INIL.

Sánchez, Víctor y Murillo, Marielos. (2004). Diaintegración, contrastividad y vocabulario de los escolares costarricenses. Káñina: Revista Artes y Letras, Universidad de Costa Rica, XXVIII (2).

Sánchez, Víctor. (2007). Estudio de lexicografía hispano-costarricense. San José: INIL.

Sequeira, Alicia. (1993). La formación de educadores en educación primaria ante la demanda del MEP. Tesis de Maestría, Universidad de Costa Rica, San José, C.R.

Ugalde, María del Carmen. (1988). El lenguaje caracterización de sus dos formas fundamentales: el código oral y el código escrito. Educación: Revista de la Universidad de Costa Rica, XII (2), 47-56. 